\title{
Modelling microwave heating of an oil palm mesocarp
}

\author{
Ming Law ${ }^{1, *}$, and Jessie Chang ${ }^{1}$ \\ ${ }^{1}$ Department of Mechanical Engineering, Faculty of Engineering and Science, Curtin University Malaysia, CDT 250, 98009, Miri, \\ Sarawak, Malaysia
}

\begin{abstract}
An oil palm mill often uses steam in sterilization and fruit detachment processes. Consequently, a large amount of wastewater is produced. To reduce the wastewater, microwave (MW) heating of oil palm fruits has been studied and positive results were reported in open literature. Nevertheless, MW heating of oil palm fruits requires proper control to avoid overheating, which deteriorates the oil quality. MW heating efficiency depends not only on the electromagnetic strength, but also on the complex permittivity of oil palm fruits. This study reports the MW heating model of an oil palm mesocarp. The three-dimensional model solved Maxwell's Electromagnetic waves equation, diffusion equation of moisture content, and heat conduction equation. The model was validated with mesocarp's experimental data of moisture content and temperatures. The electromagnetics, moisture, and temperature distributions of the mesocarp were studied. The simulation results showed that the volume- and surface-averaged temperature were similar, thus the surface temperature might be used as a good approximation to the volumetric temperature. Besides, the model also showed that radiation and convection were the main heat loss mechanisms. This validated model can be used for designing a microwave heating reactor for oil palm fruits.
\end{abstract}

\section{Introduction}

A typical oil palm fruit consists of three major parts: mesocarp (flesh of the fruit), shell and kernel. Palm oil and kernel oil can be obtained from the mesocarp and the kernel respectively. The usages of palm and kernel oils are very extensive. Palm oil is widely used in food products such as cooking oil, margarine and non-dairy creamers as well as feedstock for biofuel. In addition palm oil has a longer shelf life compared to other vegetable oils. On the other hand, palm kernel oil is a raw material for the production of non-food products such as soaps, detergents, toiletries, cosmetics and candles.

Palm and kernel oils are extracted from oil palm fruits through physical and mechanical processes in a palm oil milling process. Sterilization is the first stage of the milling process where fresh fruit bunches (FFB) are exposed to heat rendering operation with high-pressure steam operated at $15-45$ psi for about $90 \mathrm{mins}$ in horizontal cylinder autoclaves [1]. Sterilization aims to soften the fruits which allow easy detachment from the FFB and to improve the quality of fruits by deactivating the enzymatic activities. The sterilized fruits are then detached from the FFB through the threshing process in rotating thresher. Palm oil mill effluent (POME) is generated when hot steam is used in the sterilization process. According to Kamyab et al. [2], POME is one of the major environmental pollutants to contaminate the aquatic environment. In addition, POME requires an expensive and challenging treatment process before disposal [3]. Thus there is an urge to look for alternatives. Microwave (MW) heating technology could be a potential energy to replace the conventional sterilization process $[4,5]$. This is because not only the MW heating is a fast process, it also reduces energy consumption and yields a better quality of palm oils compared to the conventional sterilization process. Furthermore, POME can be eliminated as hot steam is not required in the MW heating process. In addition, the better efficiency on fruit detachment using MW heating was also observed [6].

To date, there are a limited modelling works on MW heating of oil palm fruits, compared to other materials, such as potatoes, apples and woods. Puangsuwan et al. [7] simulated the MW heating of a oil plam fruit (OPF). The model did not account for the shrinkage effects on the OPF. Law et al. [8] modelled the MW heating of oil palm kernels and the effects of OPKs arrangement in a MW reactor were studied. Recently, the effects of MW heating of $180 \mathrm{~W}$ on mechanical strength of OPKs, mesocarp and empty fruit bunches were extensively investigated by Law et al. [9]. The authors verified that MW heating could aid the detachedment of oil palm fruits from bunches. However, prolonged MW heating will cause the bunches to be harden and making the detachment more difficult. The present work is an extension of the previous work [9] and the attention is paid on the thermal behavior of oil palm mesocarp subjected to $100 \mathrm{~W}$ and $300 \mathrm{~W}$.

\footnotetext{
*Corresponding author: m.c.law@ curtin.edu.my
} 


\section{Mathematical modelling}

Figures and tables, as originals of good quality and well contrasted, are to be in their final form, ready for reproduction, pasted in the appropriate place in the text. Try to ensure that the size of the text in your figures is approximately the same size as the main text (10 point). Try to ensure that lines are no thinner than 0.25 point.

\subsection{Computational domain}

The computational domain of the model is shown as shown in Figure 1.

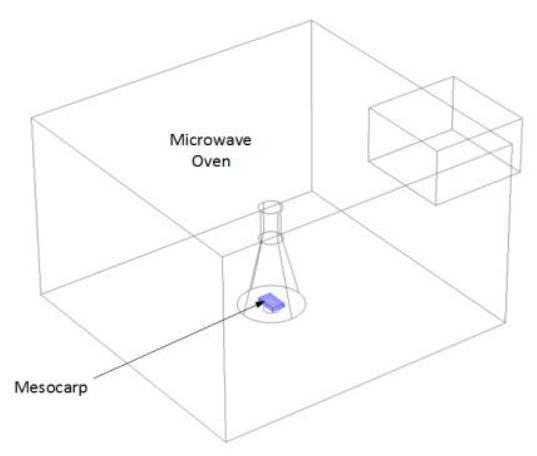

Fig. 1. The computational domain of the model.

\subsection{Assumptions}

The following assumptions were made in the current study:

1) The ambient air inside the MW oven remained in isothermal condition during the heating of mesocarp.

2) Only liquid water was considered and the effect of crude palm oil was not accounted for.

3) Moisture evaporation occurs on the mesocarp's surface only.

4) Mesocarp was assumed to be an isotropic material and the shrinkage due to the moisture lost was not considered.

\subsection{Governing equations}

The governing equations are represented as follows:

MW can be modelled by Maxwell's equation in frequency domain as

$$
\nabla \cdot(\nabla \times \mathbf{E})-\frac{\omega^{2}}{c_{o}} \mu \varepsilon \mathbf{E}=0
$$

where complex permittivity, $\varepsilon=\varepsilon_{0}\left(\varepsilon_{r}^{\prime}-j \varepsilon_{r}^{\prime \prime}\right)$.

The conservation of moisture molar concentration $\left(\mathrm{mol} / \mathrm{m}^{3}\right)$

$$
\frac{\partial c}{\partial t}=\nabla \cdot(D \nabla c)
$$

The moisture content percentage based on dry basis $(\% \mathrm{~kg} / \mathrm{kg}$ (d.b.)) can be related with $c$, via

$$
\mathrm{mc}=\frac{c \mathrm{M}_{\mathrm{H}_{2} \mathrm{O}}}{\rho} \times 100 \%
$$

where $\mathrm{M}_{\mathrm{H}_{2} \mathrm{O}}$ is the water molecular weight.

The conservation of heat energy

$$
\rho c_{p} \frac{\partial T}{\partial t}=\nabla \cdot(k \nabla T)+\dot{Q}_{M W}
$$

The MW heat source is defined as

$$
\dot{Q}_{M W}=\pi f \varepsilon_{o} \varepsilon_{r}^{\prime \prime}|\mathbf{E}|^{2}
$$

\subsubsection{Boundary conditions}

Electromagnetic boundary condition:

Two MW powers, $100 \mathrm{~W}$ and $300 \mathrm{~W}$, were applied at the inlet port of the MW oven. Intermittent heating mode was used and controlled by the oven processor, the 'on' and 'off' periods for both power level are as follows:

$$
\begin{aligned}
& P(t)=\left\{\begin{array}{cc}
100 \mathrm{~W} & t \leq 10 \mathrm{~s} \\
0 \mathrm{~W} & 10<t \leq 30 \mathrm{~s}
\end{array}\right. \\
& P(t)=\left\{\begin{array}{cc}
300 \mathrm{~W} & t \leq 13 \mathrm{~s} \\
0 \mathrm{~W} & 13<t \leq 30 \mathrm{~s}
\end{array}\right.
\end{aligned}
$$

The period of each cycle is $30 \mathrm{~s}$.

Impedance boundary condition was used for the metallic wall of the oven, i.e.

$$
\frac{1}{\mu} \mathbf{n} \times(\nabla \times \mathbf{E})-\frac{j k_{o}}{\eta} \mathbf{n} \times(\mathbf{n} \times \mathbf{E})=0
$$

where $k_{o}=\omega \sqrt{\varepsilon_{o} \mu_{o}}, \mu_{o}$ is the permeability of free space $\left(1.257 \times 10^{-6} \mathrm{H} / \mathrm{m}\right)$ and $\eta=\sqrt{\mu / \varepsilon}$.

Boundary condition of the moisture content of the oil pam mesocarp:

The moisture content was lost through evaporation and mass transfer mechanism such that,

$$
-\mathbf{n} \cdot(D \nabla c)=\dot{c}_{\text {evap }}\left(c-c_{e}\right)+h_{m}\left(c_{e}-c\right)
$$

The evaporation rate was described by a 1 st order Arrhenius rate [8],

$$
\dot{c}_{\text {evap }}=-A T \exp \left(-E_{a} / R T\right)
$$

Boundary condition of the energy conservation:

Heat losses of the mesocarp occurred through convection, radiation and moisture evaporation, such that

$$
-\mathbf{n} \cdot k \nabla T=h_{t}\left(T_{\infty}-T\right)+\dot{Q}_{r a d}+\dot{Q}_{\text {evap }}
$$

$h_{t}$ was assumed to be $5 \mathrm{~W} /(\mathrm{m} 2 . \mathrm{K})$ for natural convection in a MW oven [10]. Heat lost through radiation was described as

$$
\dot{Q}_{\text {rad }}=\gamma_{T} \sigma_{T}\left(T_{\infty}^{4}-T^{4}\right)
$$

Heat lost via evaporation was denoted as 


$$
\dot{Q}_{\text {evap }}=\dot{c}_{\text {evap }} H_{\text {evap }}
$$

The enthaphy of evaporation was as follows [11]

$$
H_{\text {evap }}=-0.0469 T^{2}-13.493 T+52202
$$

Initial conditions and input parameters:

The initial moisture content of the mesocarp was experimentally determined as $25.9 \% \mathrm{~kg} / \mathrm{kg}$ d.b. and the room temperature was $33^{\circ} \mathrm{C}$. Zero electric field strength was assumed inside the oven before the heating occurred. The input parameters of the modeling works are listed in Table 1.

Table 1. Parameters used in the model.

\begin{tabular}{|c|c|}
\hline Property & Value \\
\hline$\rho\left(\mathrm{kg} / \mathrm{m}^{3}\right)$ & $995 \mathrm{~kg} / \mathrm{m}^{3}$ \\
\hline$E_{a}(\mathrm{~J} / \mathrm{mol})$ & 43000 \\
\hline$A(1 / \mathrm{s})$ & 0.005 \\
\hline$c_{p}(\mathrm{~J} /(\mathrm{kg} . \mathrm{K}))$ & 2816 \\
\hline$k(\mathrm{~W} /(\mathrm{m} . \mathrm{K}))$ & 0.458 \\
\hline$h_{t}\left(\mathrm{~W} /\left(\mathrm{m}^{2} . \mathrm{K}\right)\right)$ & 5 \\
\hline$\varepsilon_{r}^{\prime}$ & $3.47+0.0704 \mathrm{mc}$ \\
\hline$\varepsilon_{r}^{\prime \prime}$ & $0.486+0.0402 \mathrm{mc}$ \\
\hline$h_{m}(\mathrm{~m} / \mathrm{s})$ & $\begin{array}{l}2.06 \times 10^{-6} @ 100 \mathrm{~W} \\
2.06 \times 10^{-5} @ 300 \mathrm{~W}\end{array}$ \\
\hline
\end{tabular}

\section{Results and discussion}

Figure 2 and Figure 3 show the experimental data and predicted moisture contents of the mesocarp, subject to 100 and $300 \mathrm{~W}$. Close agreement is found for both cases. Comparisons between the predicted temperature and experimental data are shown in Figure 4 and Figure 5. The tempature data was obtained at the centre of the top surface of the mesocarp using a fibre-optic temperature sensor. Good agreement is obtained for the $100 \mathrm{~W}$ case until around $\mathrm{t}=300 \mathrm{~s}$. Higher discreptancy was observed at the later stage of the MW heating as seen in Figure 4. On the other hand, good agreement was obtained for the case of MW power of $300 \mathrm{~W}$ as shown in Figure 5. To main the crude palm oil quality, it is important to keep the temperature of the mesocarp to below $100{ }^{\circ} \mathrm{C}$. Thus, when MW power of $300 \mathrm{~W}$ is used, it is advisable that the heating time should not be more than $100 \mathrm{~s}$. Nevertheless, as MW often results in non-uniform heating, it is essential to gain a more thorough understanding of the thermal response of the mesocarp before an optimum MW reactor can be designed.

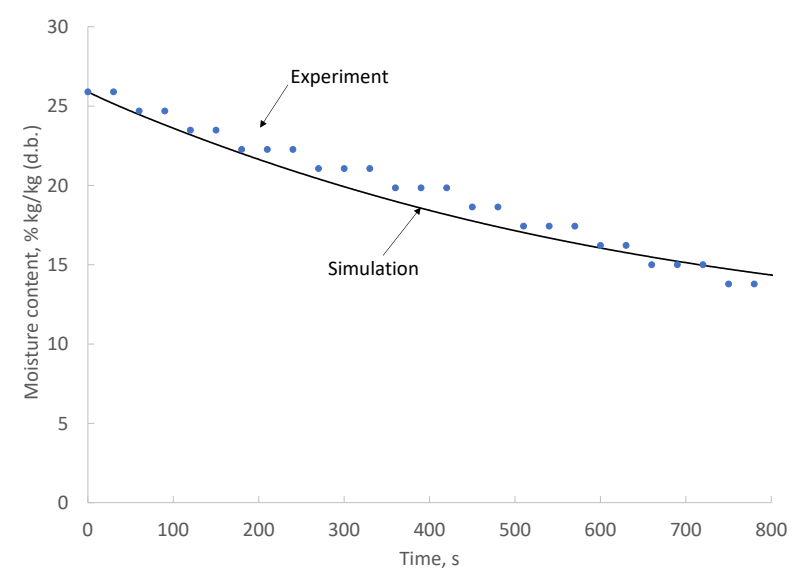

Fig. 2. Moisture content of the mesocarp sample subjected to $100 \mathrm{~W}$ MW power.

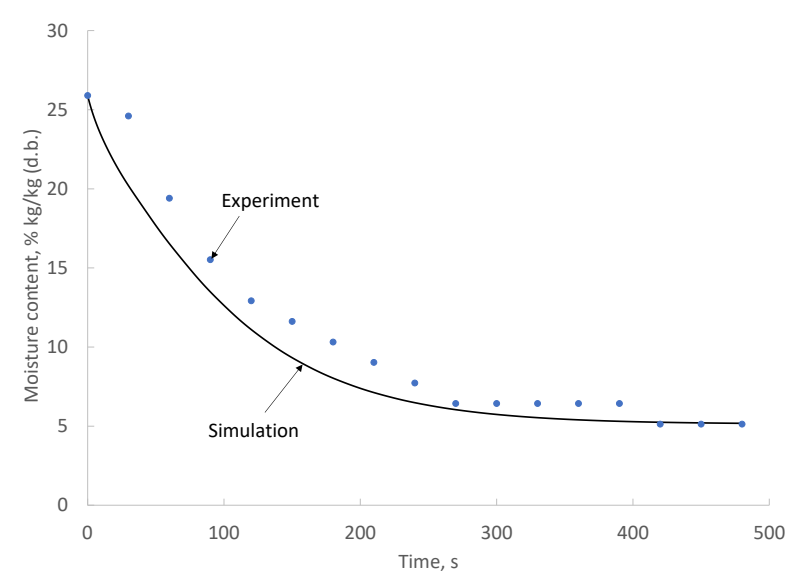

Fig. 3. Moisture content of the mesocarp sample subjected to $300 \mathrm{~W}$ of MW power.

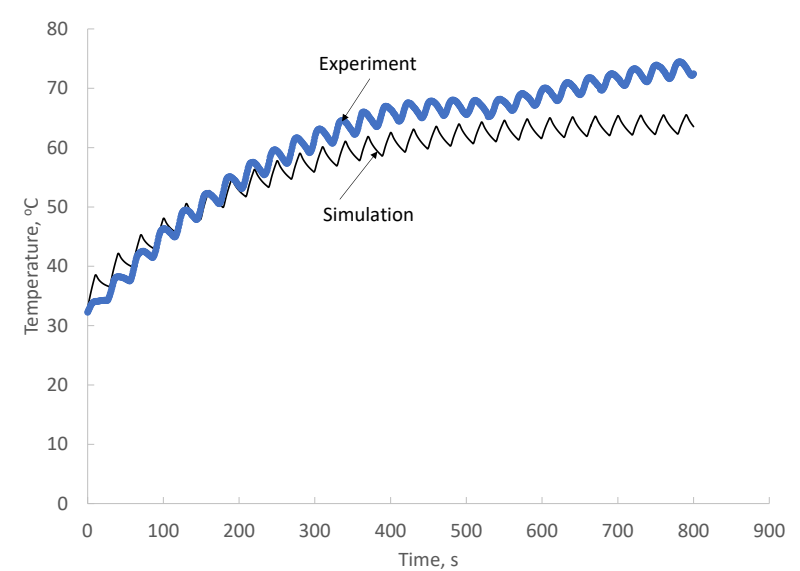

Fig. 4. Comparison between the experimental and simulationpredicted temperatures of the sample subjected to $100 \mathrm{~W}$ of MW power. 


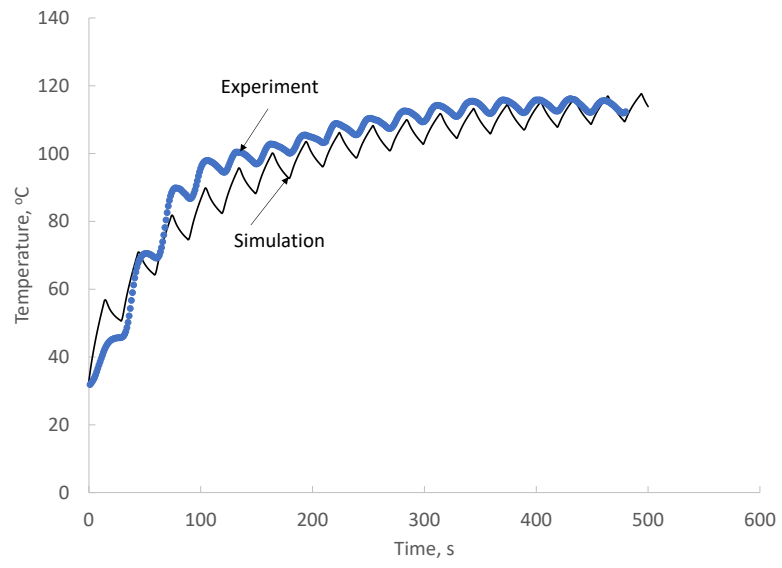

Fig. 5. Comparison between the experimental and simulationpredicted temperatures of the sample subjected to $300 \mathrm{~W}$ of MW power.

As MW heating often results in non-uniform temperature profile [8], Figure 6 shows the volume-, surface-averaged temperatures and also the maximum and minimum temperatures of the mesocarp sample subject to $300 \mathrm{~W}$. As there is only a small difference between volume- and surface-averaged temperatures, so surface temperature could be used as a good approximation for mesocarp sample. Similar readings are found for both volume- and surface-averaged temperatures because the mesocarp has a relatively high thermal conductivity. Higher difference between volume- and surface-averaged temperatures is expected if a material possesses a lower thermal conductivity. This result has an important implication in the temperature measurement because surface temperature can be obtained cheaply using an infra-red camera and the internal temperature profiles are usually obtained by using a more expensive magnetic resonance imaging (MRI) device. Hence, the result shows that surface temperature can be used as a good approximation to the volumetric-averaged temperature.

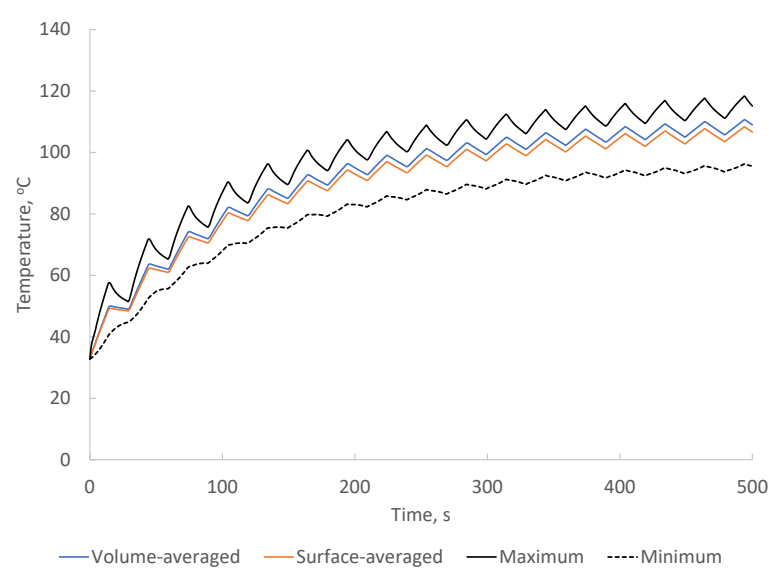

Fig. 6. Comparison between the volume- and surface-averaged temperatures of the mesocarp sample subject to a MW power of $300 \mathrm{~W}$.

In addition, the simulation shows that under $300 \mathrm{~W}$ MW irradiation, the mesocarp sample experiences a temperature difference of around $20^{\circ} \mathrm{C}$. Neverthess, the temperature of the mesocarp is affected by the MW electric field strength, E. In the current simulation, the mesocarp is placed at the centre of the MW oven but it is not at the location where the strongest electric field strength is found as can be seen in Figure 7. Hence, the temperature difference could be higher if the E field is increased. The distribution of the E field inside a MW oven is as a result of constructive and destructive interference [8]. Constructive interference results in temperature hot-spot and destructive interference causes lower temperature in a sample.

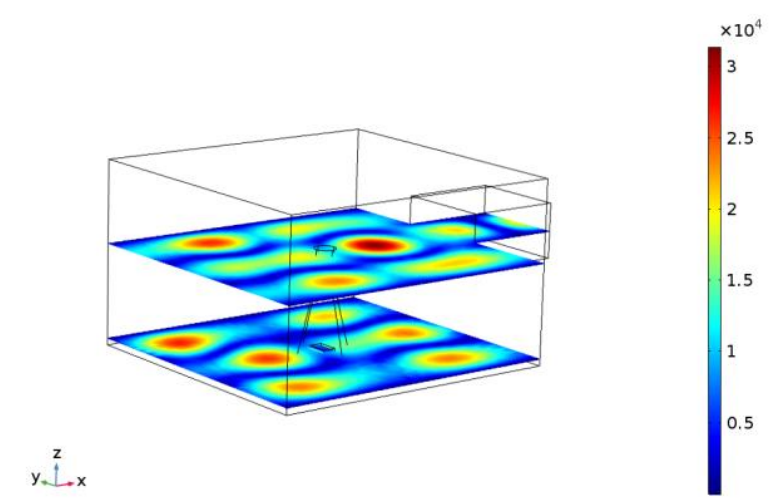

Fig. 7. The electric field strength $[\mathrm{V} / \mathrm{m}]$ distribution in the MW oven on xy plane.

Figure 8 shows the MW power density distribution in the mesocarp at $\mathrm{t}=60 \mathrm{~s}$ and $360 \mathrm{~s}$. Higher power density is found at the top part of the sample due to stronger electric field strength. As the electric field is absorbed as it passes through the sample, electric field is generally weaker at the bottom part of the sample. The MW power density absorbed is decreasing as moisture is lost during the drying process.

Figure 9 shows the internal temperature distribution of the mesocarp. The temperature and the MW power density distributions are not the same because the mesocarp's temperature is also affected by other cooling effects such as convection, radiation and evaporation. The result shows that the maximum temperature is found in the middle, internal region of the mesocarp. Comparing the experimental temperature measurement in Figure 5 and the predicted temperature profile in Figure 9, both results are in good agreement.

Figure 10 shows the surface temperature profile of the mesocarp at $\mathrm{t}=60 \mathrm{~s}$ and $360 \mathrm{~s}$. Hot spot is found at the centre of the sample. The temperature difference at $\mathrm{t}=$ $60 \mathrm{~s}$ is around $9{ }^{\circ} \mathrm{C}$ while it is around $14{ }^{\circ} \mathrm{C}$ when $\mathrm{t}=$ 360 s. We expect that the temperature non-uniformity will be more severe when a large quantity of oil palm fruits are heated inside MW oven. Hence, there is a need to carry MW heating for an actual size of an oil palm fruitlet. 

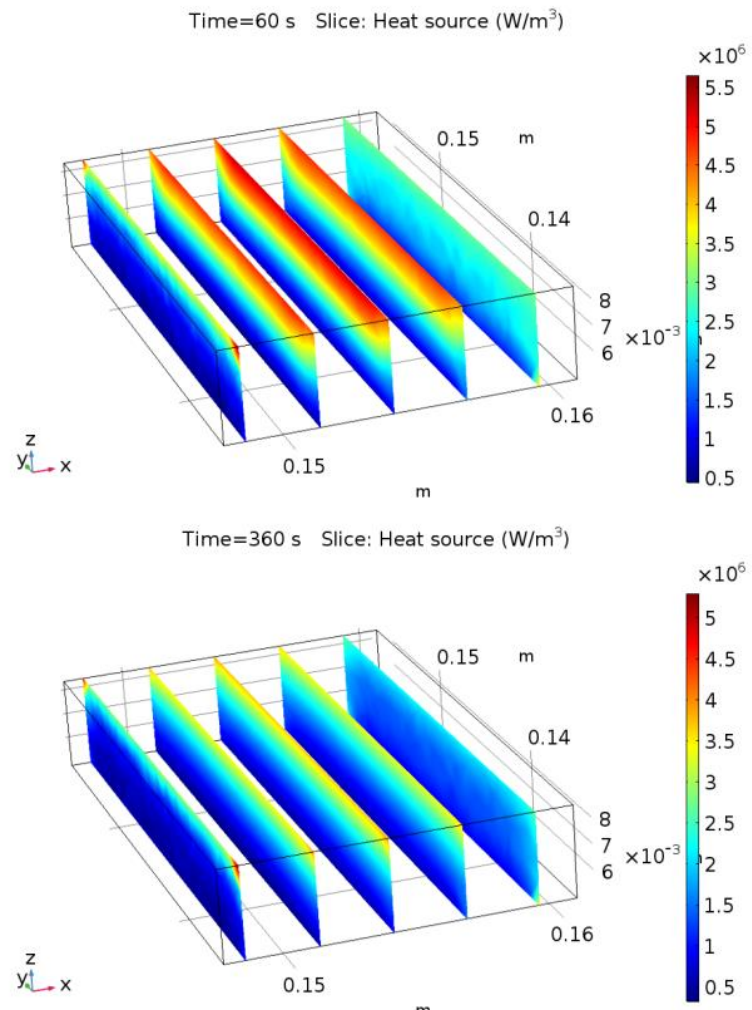

Fig. 8. The distribution of MW power density in the mesocarp sample at $\mathrm{t}=60 \mathrm{~s}$ (top) and $360 \mathrm{~s}$ (bottom)

Time $=60 \mathrm{~s}$ Slice: Temperature $(\operatorname{deg} \mathrm{C})$

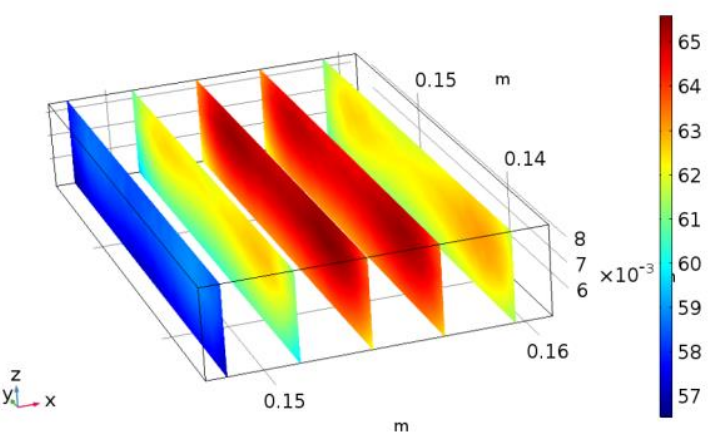

Time $=360 \mathrm{~s}$ Slice: Temperature $(\operatorname{deg} \mathrm{C})$

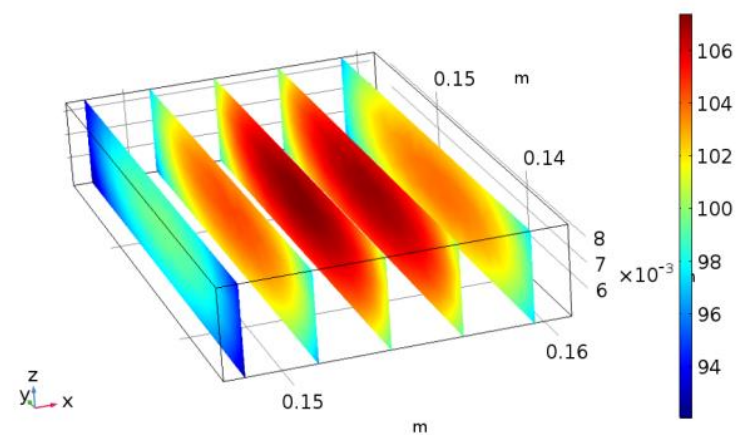

Fig. 9. Temperature distribution of the mesocarp sample at $t=$ 60s (top) and 360s (bottom)

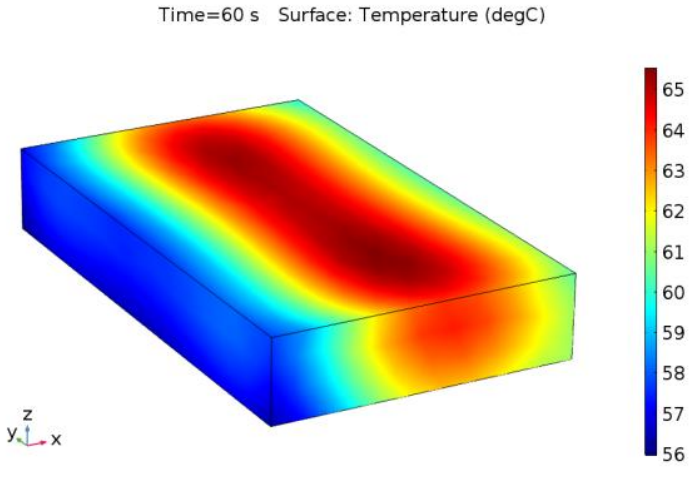

Time $=360 \mathrm{~s}$ Surface: Temperature $(\operatorname{deg} \mathrm{C})$

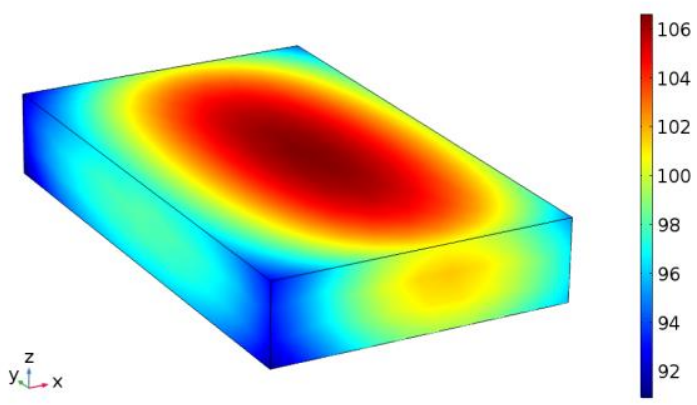

Fig. 10. Surface temperature distribution at $t=60$ s (top) and 360 s (bottom)

The heating of the mesocarp is affected by four main mechanisms, namely, MW heating, convection, evaporation and radiation. Figure 11 shows the heat power contributed by each of these mechanisms. From the result, MW heating is clearly the dominant heat source. As it depends on dielectric loss factor, which in turn depends on moisture content, hence the heat source decreases as the moisture content evaporates. The simulation result shows that convection is a main heat sink, followed by radiation and evaporation. Each of these heat sink is not more than $0.5 \mathrm{~W}$. The contribution of evaporation decreases as the mesocarp is dried.

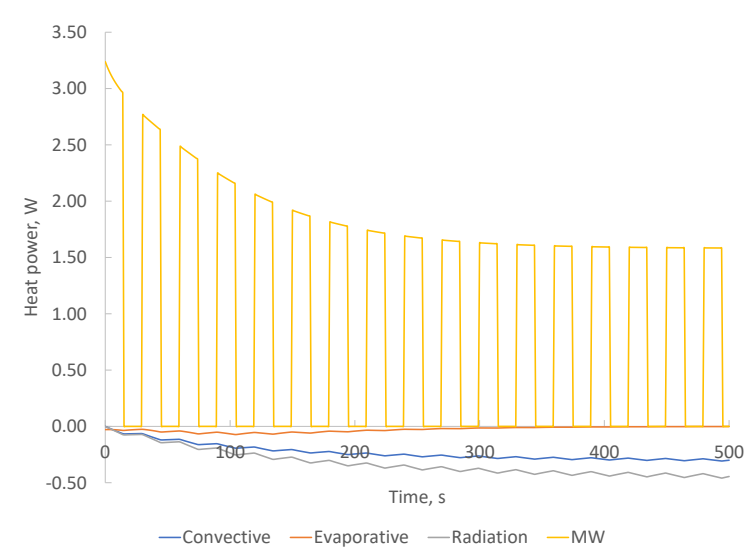

Fig. 11. Heat lost / gained by the mesocarp sample. 


\section{Conclusions}

The current study discussed the model development of an oil palm mesocarp sample subject to MW heating. The model was validated against experimental data for the moisture content and temperature history.

The model result showed that radiation and convection are dominant cooling mechanisms to reduce the surface temperature, whereby evaporation is significant when sample has high moisture content. Temperature non-uniformity increased during MW heating. Finally, surface temperature measurement can be used as an approximation for the volumetric-averaged temperature for mesocarp.

This research is supported by Malaysian Ministry of Higher Education, through the fundamental research grant schemes (FRGS/2/2014TK06/CURTIN/02/1;

FRGS/2/2014/TK06/CURTIN/03/1).

\section{References}

1. K. Sivasothy, M. Rohaya, International Planters Conference, Kuala Lumpur (2000)

2. H. Kamyab, M. Din, A. Keyvanfar, M. Majid, A. Talaiekhozani, A. Shafaghat, C. Lee, L. Shiun, H. Ismail, Energy Procedia, 75, 2400-2408, (2015)

3. Y. Madaki, L. Seng, 2013, IJSET, 2, 1138 - 1155, (2013)

4. C. Vincent, R. Shamsudin, A. Baharuddin, J Food Eng, 143,123 - 131, (2014)

5. M. Chow, A. Ma, J Microw Power Electromagn Energy, 40, pp. 165 - 173, (2007)

6. N. Sukaribin, K. Khalid, Ind Crops Prod., 30, 179183, (2009)

7. K. Puangsuwan, M. Chongcheawchamnan, C. Tongurai, J Microw Power Electromagn Energy, 49, 100-111, (2015)

8. M. Law, E. Liew, S. Chang, Y. Chan, C. Leo, Appl Therm Eng., 98, 702-726, (2016)

9. M. Law, J. Chang, Y. Chan, D. Pui, K. You, Drying Technol, (2018), DOI: $\underline{10.1080 / 07373937.2018 .1439057}$

10. P. Verboven, A. Datta, N. Anh, N. Scheerlinck, B. Nicolaï, J Food Eng, 59, 181-190, (2003)

11. C. Hii, C. Law, C.L., Law, M.C., 2013, Appl Therm Eng., 54, 264-271, (2013) 